\title{
Impedance spectroscopy for monosaccharides detection using responsive hydrogel modified paper-based electrodes
}

C. M. Daikuzono a,b, C. Delaneya, H. Tesfaya, L. Florea ${ }^{a *}$, O. N. Oliveira, Jr.b*, A. Morrin ${ }^{\mathrm{a}}$ and D. Diamond ${ }^{\mathrm{a}}$

Insight Centre for Data Analytics, National Centre for Sensor Research, School of Chemical Sciences, Dublin City University, Ireland

Instituto de Física de São Carlos, Universidade de São Paulo, Brazil

Electronic Supplementary Information (ESI) available: [details of any supplementary information available should be included here]. See DOI: $10.1039 / \mathrm{x} 0 \mathrm{xx} 00000 \mathrm{x}$

Herein we present a novel sensor for the detection of monosaccharides (e.g. glucose, fructose) in solution, using electrical impedance spectroscopy. The sensor is based on carbon interdigitated electrodes, printed on paper using screen printing. The surface of the electrodes was modified with a thin layer of hydrogel containing acrylamide copolymerised with $20 \mathrm{~mol} \% \quad 3$ (Acrylamido)phenylboronic acid (PBA). It was observed that the hydrogel layers containing $20 \mathrm{~mol} \%$ PBA swell considerably in the presence of glucose and fructose. This in turn changes the measured impedance across the electrodes, making it a suitable sensor for the quantitative detection of saccharides. We investigated the impedance and capacitance variations with different concentrations of glucose and fructose $(0-5 \mathrm{mM})$ in aqueous phosphate buffer solutions. Variations in impedance were attributed to changes in the dielectric properties of the hydrogel under an applied electric field, due to swelling of the hydrogel layer induced by uptake and binding of sugar molecules to the boronate species within the gel. Impedance measurements at $1 \mathrm{kHz}$ demonstrated that hydrogel swelling leads to an increased mobility of ions within the swollen hydrogel layer. The impedance decreased with increasing sugar concentration and the relative capacitance curves are markedly different for fructose and glucose, as the hydrogel exhibits greater swelling in the presence of fructose than glucose over the same concentration range. As the proposed sensor was shown to be suitable for the detection of glucose at concentration levels found in human sweat, future work will focus on the incorporation of these modified paper-based electrodes into wearable skin patches for non-invasive sugar monitoring in sweat.

\section{Introduction}

According to the World Health Organization, diabetes is a chronic disease, characterized by elevated levels of blood glucose. There are 422 million adults suffering from diabetes in the world today. Diabetes can cause serious complications for health such as kidney failure, heart attack and blindness. For this reason there is an ever-growing interest in the development of sensor devices for monitoring glucose through both invasive1 and non-invasive2 methods. Although minimally-invasive, common self-monitoring methods of blood glucose using finger (or forearm, thigh) pricking, or subcutaneous implantable sensors, continue to bring discomfort to the patients. Currently, finger pricking remains the most frequent method used to monitor glycaemia in diabetics but due to its non-continuous approach, episodes of hyper or hypo- 
glycaemia can be overlooked. In contrast, non-invasive methods are advantageous as they are painless and require samples of body fluids, such as sweat,3, 4 tears 5 or saliva6. In order to be functional, these sensors need to have significantly higher sensitivity, as the glucose levels in these matrices are typically lower than the ones found in blood: glucose levels in blood within 4-8 $\mathrm{mM}$ (72-144 mg.dL-1)7 while for sweat 0.28-1.11 mM (5-20 mg.dL-1).8 In an attempt to overcome such challenges as limit of detection, wearability, cost and sensitivity, the scientific community has responded en masse, with the number of papers published by the research community on the topic per year increasing from about 500 in 2006 to about 1500 in 2015 (Source: Web of Science). Detection of glucose within distinct concentration ranges can be made using various techniques, including electrochemical 9, 10, optical11, 12 and impedance spectroscopy 13-16 methods, where the sensing mechanism generally employs the use of the glucose oxidase enzyme4,12, 14, 15, 17 or sugar binding molecules such as boronic acid derivatives10. Boronic acids (BA) are used as receptors in a large number of chemosensors and they represent a good alternative to enzymes for monosaccharides sensing. The reversible covalent interaction of boronic acids with diol groups, such as those present in saccharides, offers the possibility of sugar detection at sub-mM concentrations. Boronic acids are composed of an electron deficient boron atom with two attached hydroxyl groups18, which makes these molecules Lewis acidic. This Lewis acidic behaviour accounts for the strong interactions with electronegative hydroxyl groups on sugar molecules18-23 and thereby allows for the recognition of saccharides in the physiological $\mathrm{pH}$ range. In solution, BA derivatives have been used in tandem with fluorophores, either through covalent or non-covalent binding, for optical (fluorescence) detection of sugars. The conformational change around the boron atom upon sugar binding affects the interaction between the BA and the fluorescent moiety, thereby changing the fluorescence of the system. Non-covalent doping of soft polymeric platforms such as contact lenses, with fluorescent BA solutions has been proposed by Badugu et al.5 for the development of smart contact lenses, which could be used for glucose detection in the range of $50-100 \mu \mathrm{M}$, relevant for ocular fluid. Leaching of the sensor over time would constitute a major disadvantage of this approach. Covalent incorporation of BA derivatives into polymeric systems or field effect transistors has also been proposed, in order to fabricate integrated BA sensors with improved stability. In this regard, carbon nanotubes functionalized with pyrene1-boronic acid have been used for the fabrication of a carbon nanotube based field effect transistor for glucose sensing in the clinically relevant range for blood or saliva.24 Phenylboronic acid (PBA) modified graphene oxide, was proven efficient for use in the differential pulse voltammetry detection of fructose, mannose and glucose, with a $0.8 \mathrm{mM}$ detection limit for glucose 25 while BA functionalised gold electrodes were used for the electrochemical impedance detection of four types of monosaccharide; including glucose, sorbitol, mannitol, and fructose. 26

Incorporation of BA derivatives into hydrogel materials has also been employed for sugar detection by several research groups, as the interaction between the boronic acid and the sugar induces a change in hydrogel volume, which is modulated by sugar concentration. Diameter measurements of the hydrogel 
swelling can then be converted into sugar concentration.27 Such measurements lack the precision required for sugar sensors. Nevertheless, differences in swelling can in turn cause changes in the optical or electrical properties of the hydrogel, enabling various means of detection with significantly improved accuracy. Tierney et al.28 developed an optical sensor for glucose detection using PBA incorporated in a hydrogel as a Fabry-Perot cavity at the end of an optical fiber. The optical length of the gel can be determined through an interface wave formed by reflection from light in fiber-gel and gel-solution interfaces. They demonstrated that the gel had a linear swelling response in aqueous solution with $2.5 \mathrm{mM}$ carbohydrates which was determined to be $-1760 \mathrm{~nm} \mathrm{mM}$ 1 for glucose. Introduction of a crystalline colloidal array embedded in a similar PBA hydrogel matrix allows for volume changes induced by PBA-glucose interactions to be converted in a wavelength shift of the Bragg diffracted light.29

Just recently, a PBA hydrogel was used in a MEMS capacitive transducer, in order to measure the changes in the dielectric properties of the hydrogel upon sugar binding 30. In this case, the PBA hydrogel was sandwiched between two parallel thin-film micro-electrodes fabricated by photo-lithography. It was found that the effective capacitance of the sensor could be measured and related to the glucose concentration. However, the absolute change in effective capacitance in the low glucose concentration region (0-40 mg.dL-1) was less than $0.5 \mathrm{pF}$, with no intermediary concentrations analysed, making such sensor probably unsuitable for sweat analysis.

Herein, we present a low-cost, disposable alternative to this approach with improved sensitivity at low sugar concentrations. The sensor consists of interdigitated carbon screen-printed electrodes on paper coated with a thin hydrogel layer containing PBA. Changes in the dielectric properties of the hydrogel upon sugar interaction are measured using impedance spectroscopy. Capacitance and impedance variations with different concentrations of glucose and fructose, in buffered aqueous solutions, were investigated in the $0-5 \mathrm{mM}$ concentration range. Advantages of this type of device include easy manipulation, low cost and scalability due to electrode fabrication techniques (e.g. screen printing). Moreover, the porous/fibrous structure of the paper allows for direct immobilization of the hydrogel, eliminating the use of semipermeable membranes that are otherwise required to hold the glucose-sensitive material 1 in place. The sensitivity and operational range of the sensor, together with the paper support platform, permit further development of this sensor towards the realisation of a microfluidic paper-based analytical device for sugar analysis in sweat.

\section{Experimental \\ Materials}

Acrylamide 99\% (AAm), N,N'-Methylenebis(acrylamide) 99\% (MBIS), 2Hydroxy-2-methylpropiophenone 97\% (HMPP), 3-(Acrylamido)phenylboronic acid 98\% (PBA), D-(-)-Fructose, D-(+)-glucose, dimethyl sulfoxide (DMSO) and filter paper Watman (grade 158 x $68 \mathrm{~cm}$ and pore size $11 \mu \mathrm{m}$ ) were purchased from Sigma-Aldrich, Ireland and used as received. Carbon graphite ink was obtained from GWENT GROUP Inc. Pontypool, UK (product code C2030519P4). All aqueous solutions were prepared with ultrapure water $(18.2 \mathrm{M} \Omega \mathrm{cm}$ resistivity, Millipore Milli-Q). 


\section{Fabrication of Carbon Interdigitated Electrodes}

The carbon interdigitated electrodes $(15 \mathrm{~mm} \times 10 \mathrm{~mm}, 10$ digits with $1 \mathrm{~mm}$ of spacing) were screen-printed on paper using a DEK 248 screen printer. Following printing, the electrodes were dried in an oven at $50^{\circ} \mathrm{C}$ for $40 \mathrm{~min}$.

Synthesis and Swelling Studies of p(AAm-co-PBA) Free-Standing Hydrogels Swelling assays for the $\mathrm{p}(\mathrm{AAm}-\mathrm{Co}$-PBA) hydrogels were performed using various concentrations of glucose and fructose. The hydrogel was prepared by mixing $0.25 \mathrm{~g} \mathrm{AAm}, 1 \mathrm{~mol} \% \mathrm{MBIS}, 1 \mathrm{~mol} \% \mathrm{HMPP}$ and $20 \mathrm{~mol} \%$ PBA, respectively, dissolved in $500 \mu \mathrm{L}$ water/DMSO (1:1). $200 \mu \mathrm{L}$ of this cocktail was placed into a polydimethylsiloxane (PDMS) mould with $10 \mathrm{~mm}$ diameter wells and exposed to UV light (CL-1000 Ultraviolet crosslinker UVP) for $30 \mathrm{~min}$ to allow for polymerization of the hydrogel. Smaller circular hydrogels (4 $\mathrm{mm}$ diameter) were cut from the cured hydrogel using a manual punch (4 $\mathrm{mm}$ diameter) and each disc was weighed (Wdry). Following this, the hydrogel discs were hydrated in a phosphate buffer ( $\mathrm{pH}$ 7.4) only, or in buffer containing various concentrations of glucose or fructose for $24 \mathrm{~h}$ at room temperature. After this period of time, any surface water was carefully removed from each disc, and the disc reweighed $(\mathrm{Ww})$ to calculate the swelling ratio using equation 1.

Swelling Ratio $=W w-W d r y w d r y$

(Eq. 1)

\section{Electrode Functionalization Protocol and Analysis}

In order to study the influence of hydrogel functionalization on measured impedance, an increasing number of hydrogel layers (0-5) were deposited on top of the electrodes by drop casting. For this, $10 \mu \mathrm{L}$ of the polymerisation cocktail (without PBA) was placed on top of the electrodes and polymerised for $30 \mathrm{~min}$ with UV light for each layer deposited. Between each hydrogel layer deposition, the impedance and hence capacitance of the electrodes were measured in deionized water. Separately, Scanning Electron Microscopy (SEM) images of the electrodes were collected after the deposition of each hydrogel layer. The electrodes fabricated for all sugar sensing experiments were modified with just 1 layer of hydrogel using the same functionalization protocol.

\section{Electrical Impedance Spectroscopy (EIS)}

The electrical impedance spectroscopy measurements were performed using an impedance analyser (Solartron 1260). The impedance spectrum was acquired by applying an AC signal of $20 \mathrm{mV}$ across the interdigitated electrodes over a frequency range from 0.1 to $10 \mathrm{MHz}$ with 5 points per decade. The interdigitated electrode was initially immersed in PBS buffer for 12 min to ensure stabilization of the electrical double layer. For subsequent sugar solutions the system was allowed to stabilize for 6 min between measurements. Different concentrations (0-5 mM) of glucose or fructose, prepared in phosphate buffer ( $\mathrm{pH} 7.4)$, were monitored through the measured capacitance value at $1 \mathrm{kHz}$. The relative capacitance $(\Delta C)$ was calculated using equation 2 , where $C s$ is the device capacitance in the presence of sugar in phosphate buffer and $C b$ is the device capacitance in buffer solution.

$\Delta \mathrm{C}=\mathrm{Cs}-\mathrm{Cb}$ 


\section{Results and discussion}

\section{Free-Standing p(AAm-co-PBA) Hydrogel Characterization}

A hydrogel matrix containing copolymerized PBA constitutes a highly suitable non-enzymatic sensing unit for determination of sugar concentration, as PBA has a high affinity towards diol moieties 31. In hydrogels containing PBA, at physiological pH 7.4, the majority of PBA would remain in the uncharged form, as the pKa of the PBA is approximately 8.6 32. In the presence of sugar molecules however (e.g. glucose, fructose), the pKa is lowered and thus the concentration of the anionic boronate species in the polymer increases upon binding. The increase of a bulk negative charge in the hydrogel, upon generation of the boronate groups, creates an osmotic swelling force causing hydrogel swelling 1 , $25,27,29$.

We first investigated the behaviour of small hydrogel discs (4 $\mathrm{mm}$ diameter) of p(AAm-co-PBA) with 20 mol \% PBA in phosphate buffer ( $\mathrm{pH} 7.4$ ) in the presence of $20 \mathrm{mM}$ glucose and $20 \mathrm{mM}$ of fructose, respectively. Figure 4 shows that the hydrogels expand considerably in the presence of sugar, with larger volumetric changes for fructose, in comparison with glucose solutions. The orientation and relative position of hydroxyl groups in the sugar molecule determine the strength of binding of BA with saccharides. PBA has been shown to exhibit a binding constant for fructose which is $\sim 40$ times higher than that for glucose, under physiological conditions 33.

Hydrogel swelling increases with the concentration of sugar, as seen in Figure 5, in the case of glucose and fructose solutions of 0-5 mM, in PBS buffer.

\section{p(AAm-co-PBA) Hydrogel Layer for Electrode Coating}

Figure 6 shows representative SEM images of hydrogel layers on top of the screen-printed interdigitated electrodes on paper, which highlights the increasing thickness with the number of layers applied. This increase in thickness is not desirable for the impedance sensor, as the hydrogel material is insulating. Figure 7 shows that the impedance of an electrode coated with hydrogel (without PBA) increases with the number of layers. Therefore, following this screening measurement, further experiments were made by coating the interdigitated electrodes with one hydrogel layer only. A single, thin layer allows for faster diffusion through the gel, increased ion mobility and hence more rapid equilibrium swelling times.

\section{Impedance and Capacitance Analysis}

The interaction between the sugar and the PBA group induces changes in the dielectric properties of the hydrogel film, which can be measured by impedance spectroscopy. Different concentration of sugar in solution induce thin layer hydrogel swelling, leading to increased diffusion of ion species and thus increased current flow that can be measure through capacitance or impedance changes. Figure S1 depicts capacitance behaviour for varied glucose concentration (5-30 mM) at different PBA compositions (5 mol\% and $20 \mathrm{~mol} \%$ ) in the hydrogel. Increasing the concentration of PBA in the hydrogel allowed for greater numbers of glucose molecules to bind, thereby inducing swelling and 
increasing the change in capacitance. This variation in the dielectric properties of the hydrogel can be detected by measuring capacitance at $1 \mathrm{kHz}$.

The impedance spectra in Figure 8, using a single layer of p(AAm-co-PBA) hydrogel (20 mol\% PBA composition) on the electrode, shows variations across different concentrations of glucose or fructose, across the full frequency range investigated. As the sugar molecules diffuse into the hydrogel matrix, inducing swelling, the mobility of ions within the hydrogel increases thereby changing the electrical properties of the hydrogel_ENREF_3434. It was observed that frequencies in the $1 \mathrm{kHz}$ range exhibited the greatest difference in impedance when exposed to varied sugar concentrations. By measuring the impedance at 1 $\mathrm{kHz}$, a monotonic decrease with increasing sugar concentrations can be observed, as shown in the insets of Figure 8 for both glucose and fructose. Such a decrease in impedance may be related to increased ion-mobility within the swollen hydrogel.

Both sugars tested showed a linear increase in the range of sugar concentrations from 0.2 to $1.2 \mathrm{mM}$ and a smaller variation was seen in the case of the bare electrode. For the reproducibility studies, special attention has been dedicated to fabricate reproducible bare electrodes. Their response to buffer solution has been investigated prior to hydrogel functionalization. This ensures that any variations in sensor response are not caused by the intrinsic characteristic of the carbon printed electrodes. The response of the sensor coated

with p(AAm-co-PBA) hydrogel (with $20 \mathrm{~mol} \% \mathrm{PBA}$ ) is greater for fructose, compared to glucose. It is therefore possible to differentiate between glucose and fructose based on the slope of the sensor response. It was observed that for concentrations above $2 \mathrm{mM}$, there was a tendency to saturate the signal with the capacitance change becoming practically independent of sugar concentration. This is likely due to the hydrogel reaching its maximum swelling capacity. A more detailed analysis was performed with the capacitance at $1 \mathrm{kHz}$, with the capacitance change $(\Delta C)$ being plotted against the sugar concentration in Figure 9. As expected from the larger hydrogel swelling caused by fructose, the changes in capacitance are more significant for fructose than for glucose at the same concentration. The sensor is adequate for detecting small sugar concentrations, e.g. from 0.2 to $1.2 \mathrm{mM}$ (Figure 9B), making this sensor relevant for human sweat analysis in which the glucose concentration is within the 0.28 to $1.11 \mathrm{mM}$ range.

\section{Conclusions}

We have presented hydrogel modified paper-based electrodes for the detection of monosaccharides (e.g. glucose, fructose) in solution, using electrical impedance spectroscopy. The modified electrodes were realised by coating carbon screen-printed interdigitated electrodes with a thin layer of synthetic hydrogel film containing $20 \mathrm{~mol} \%$ phenylboronic acid. As sugar molecules permeate into the hydrogel they bind to the boronic acid moieties, resulting in the formation of boronate groups. This binding leads to an increase in the bulk negative charge of the hydrogel and, through an osmotic swelling force, causes the hydrogel to swell. This sugar-induced swelling increases the mobility of ions within the swollen hydrogel thereby changing its electrical properties34. Experimental results showed that impedance and capacitance variations of the device, in the measurement frequency range, responded consistently to sugar 
concentrations from 0 to $5 \mathrm{mM}$, in aqueous phosphate buffer solutions. Impedance measured at a frequency of $1 \mathrm{kHz}$ shows a decrease with increasing sugar concentration. Additionally, the relative capacitance changes observed are markedly different for fructose and glucose, as the hydrogel exhibits greater swelling in the presence of fructose than glucose over the same concentration range. However, with this simple sensor, quantification of different types of sugars present in one sample is not possible, and for this scope an array of differently functionalised interdigitated electrodes and principal component analysis (PCA) is envisioned. As the proposed sensor was shown to be suitable for the detection of glucose at concentration levels found in human sweat, future work will focus on the incorporation of these modified paper-based electrodes into disposable wearable skin patches for non-invasive sugar monitoring in sweat. Future development of the sensor could consider external interferences from sweat, together with varied electrolyte concentration and sweat rates, which could potentially be overcome by using a multisensor approach. This would allow for quantification of external influences such as ion concentration and hydration of the hydrogel coating.

\section{Acknowledgements}

This project has been funded by Science Foundation Ireland under the Insight initiative, grant SFI/12/RC/2289 and Coordenação de aperfeiçoamento de pessoal de nível superior (CAPES). CD and DD also acknowledge the European Union's Seventh Framework Programme for research, technological development, and demonstration, through the NAPES project grant agreement no. 604241.

\section{References}

1. X. Huang, C. Leduc, Y. Ravussin, S. Li, E. Davis, B. Song, D. Li, K. Xu, D. Accili and Q. Wang, Lab on a Chip, 2014, 14, 294.

2. C.-F. So, K.-S. Choi, T. K. Wong and J. W. Chung, Medical devices, 2012, 5, 45. 3. W. Gao, S. Emaminejad, H. Y. Y. Nyein, S. Challa, K. Chen, A. Peck, H. M. Fahad, H. Ota, H. Shiraki and D. Kiriya, Nature, 2016, 529, 509.

4. O. Olarte, J. Chilo, J. Pelegri-Sebastia, K. Barbé and W. Van Moer, 35th Annual International Conference of the IEEE Engineering in Medicine and Biology Society (EMBC), 2013, 1462.

5. R. Badugu, J. R. Lakowicz and C. D. Geddes, Journal of Fluorescence, 2003, 13, 371-374.

6. S. Malik, R. Khadgawat, S. Anand and S. Gupta, SpringerPlus, 2016, 1.

7. D. Rodbard, Diabetes technology \& therapeutics, 2016, 18, S2-3.

8. O. Mickelsen and A. Keys, Journal of Biological Chemistry, 1943, 149, 479.

9. J. Wang, Chemical reviews, 2008, 108, 814-825.

10. J. Anzai, Materials Science and Engineering: C, 2016, 67, 737.

11. Y. Kim, G. Jang, D. Kim, J. Kim and T. S. Lee, Polymer Chemistry, 2016, 7, 1907.

12. H. Wang, J. Yi, D. Velado, Y. Yu and S. Zhou, ACS applied materials \& interfaces, 2015, 7, 15735.

13. M. L. Moraes, L. Petri, V. Oliveira, C. A. Olivati, M. C. F. de Oliveira, F. V. Paulovich, O. N. Oliveira and M. Ferreira, Sensors and Actuators B: Chemical, 2012, 166, 231.

14. S.-Y. Huang, C.-M. Chou, T.-H. Chen, P.-C. Chiou, V. K. Hsiao, C. T.-S. Ching and T.-P. Sun, Journal of The Electrochemical Society, 2014, 161, B102. 
15. N. Mac Kenna, P. Calvert and A. Morrin, Analyst, 2015, 140, 3003.

16. A. Tura, S. Sbrignadello, S. Barison, S. Conti and G. Pacini, Biophysical chemistry, 2007, 129, 235.

17. G. Matzeu, L. Florea and D. Diamond, Sensors and Actuators B: Chemical, 2015, 211, 403.

18. R. Badugu, J. R. Lakowicz, and C. D. Geddes, Anal. Chem., 2004, 76, 610.

19. H. Fang, G. Kaur and B. Wang, Journal of Fluorescence, 2004, 14, 481.

20. R. Badugu, J. Lakowicz and C. D. Geddes, Bioorganic and Medicinal Chemistry, 2005, 13, 113.

21. R. Badugu, J. Lakowicz and C. D. Geddes, Analyst, 2004, 129, 516.

22. R. Badugu, J. Lakowicz and C. D. Geddes, Journal of Fluorescence, 2004, 14, 617.

23. Z. L. Xin Wu, X.-X. Chen, J. S. Fossey, T. D. James and Y-B. Jiang, Chemical Society reviews, 2013, 42, 8032.

24. M. B. Lerner, N. Kybert, R. Mendoza, R. Villechenon, M. A. B. Lopez and A. C. Johnson, Applied Physics Letters, 2013, 102, 183113.

25. Q. Wang, I. Kaminska, J. Niedziolka-Jonsson, M. Opallo, M. Li, R. Boukherroub and S. Szunerits, Biosensors and Bioelectronics, 2013, 50, 331.

26. Y. Ma and X. Yang, Journal of Electroanalytical Chemistry, 2005, 580, 348352.

27. A. Kim, S. K. Mujumdar and R. A. Siegel, Chemosensors, 2013, 2, 1.

28. S. Tierney, S. Volden and B. T. Stokke, Biosensors and Bioelectronics, 2009, 24, 2034.

29. C. Zhang, M. D. Losego and P. V. Braun, Chemistry of Materials, 2013, 25, 3239.

30. J. Shang, J. Yan, Z. Zhang, X. Huang, P. Maturavongsadit, B. Song, Y. Jia, T. Ma, D. Li and K. Xu, Sensors and Actuators B: Chemical, 2016, 237, 992.

31. X. Wu, Z. Li, X.-X. Chen, J. S. Fossey, T. D. James and Y.-B. Jiang, Chemical Society Reviews, 2013, 42, 8032.

32. L. Babcock and R. Pizer, Inorganic Chemistry, 1980, 19, 56.

33. J. P. Lorand and J. O. Edwards, The Journal of Organic Chemistry, 1959, 24, 769.

34. A. Kikuchi, K. Suzuki, O. Okabayashi, H. Hoshino, K. Kataoka, Y. Sakurai and T. Okano, Analytical chemistry, 1996, 68, 823.

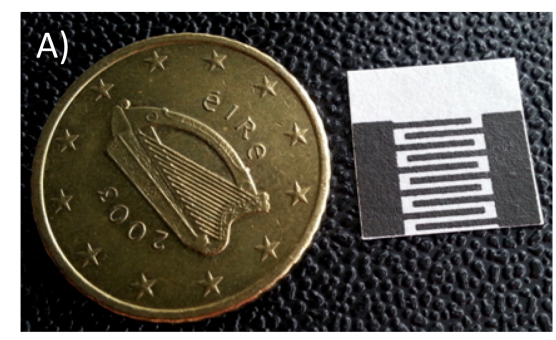

B)

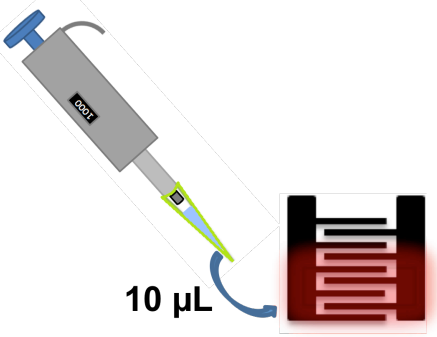

C) UV light $30 \mathrm{~min}$

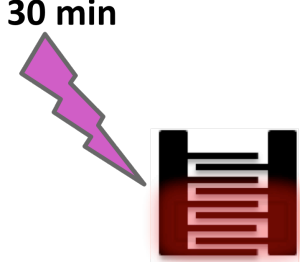

Fig. 1. Coating of screen-printed carbon interdigitated electrodes with p(AAmco-PBA) hydrogel layer; A) Photo of screen-printed carbon interdigitated electrodes before functionalization; B) Schematic of cocktail deposition on top of the electrodes by drop-casting and C) hydrogel photo-polymerisation protocol. 


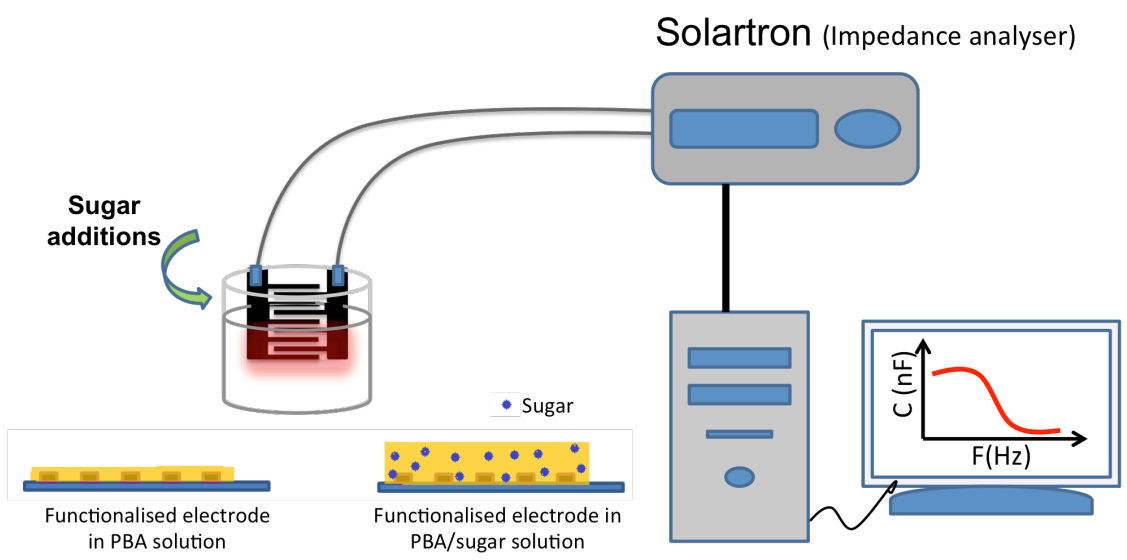

Fig. 2. Schematic of the experimental setup for impedance detection of monosaccharides using $\mathrm{p}(\mathrm{AAm}-\mathrm{co}-\mathrm{PBA})$ hydrogel functionalized electrodes.
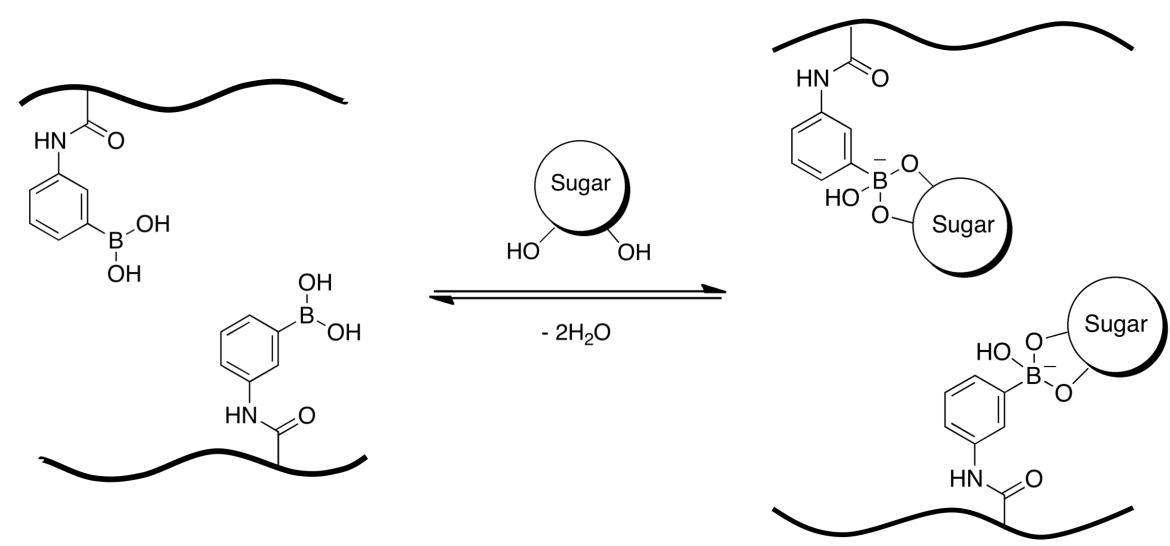

Fig. 3. Schematic of the p(AAm-co-PBA) polymer chain showing the structure of copolymerized 3-acrylamidophenylboronic acid (PBA) and its reversible binding with sugar molecules.
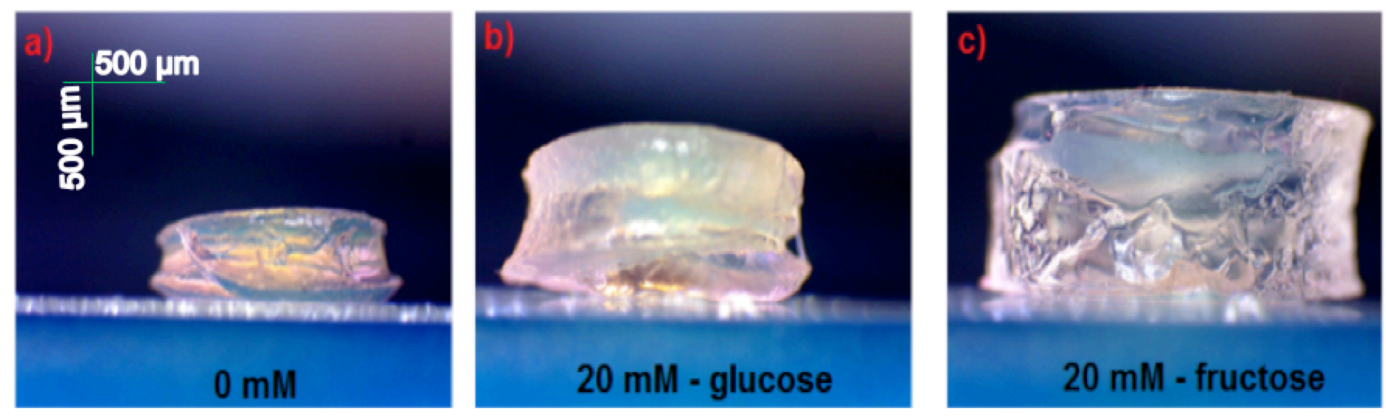

Fig. 4. Microscope images of p(AAm-co-PBA) hydrogels with $20 \% \mathrm{~mol}$ PBA, when immersed in (a) PBS buffer, (b) $20 \mathrm{mM}$ glucose in PBS and (c) $20 \mathrm{mM}$ fructose in PBS. 


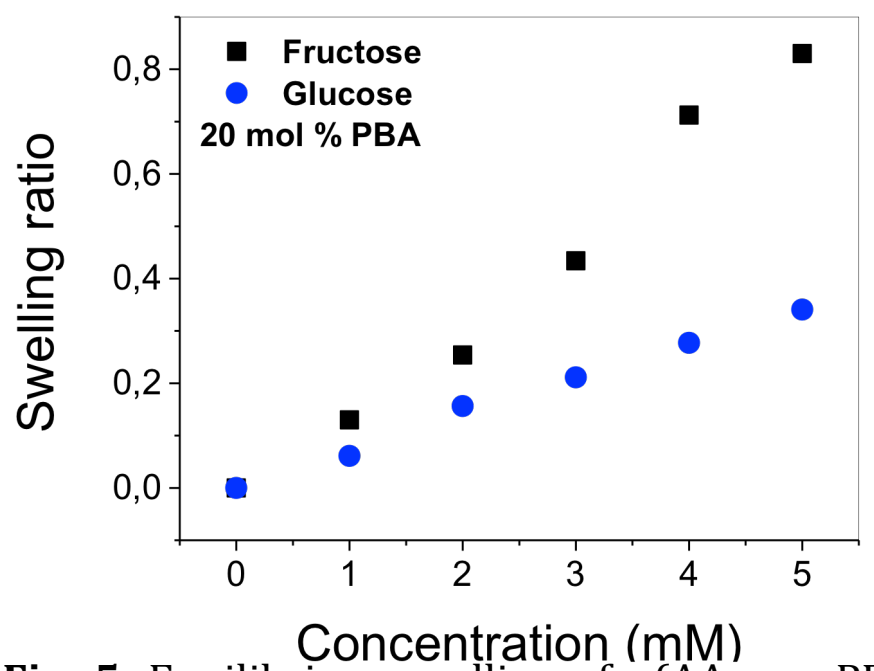

Fig. 5. Equilibrium swelling of p(AAm-co-PBA) hydrogels with $20 \% \mathrm{~mol}$ PBA in buffer solution at different glucose and fructose concentrations.

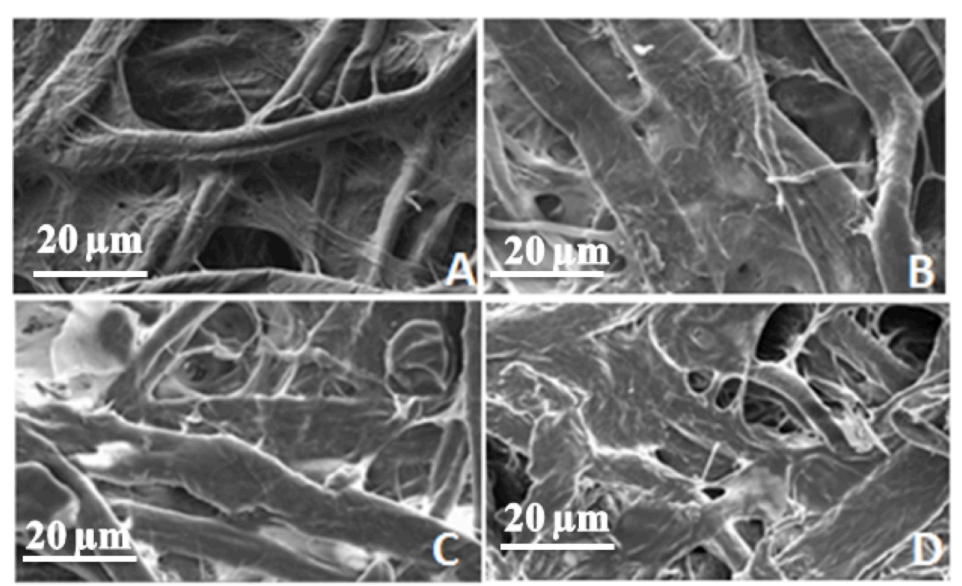

Fig. 6. SEM images of screen-printed carbon on paper before (A) and after being coated with 1 (B), 3 (C) and 5 (D) hydrogel layers, respectively.

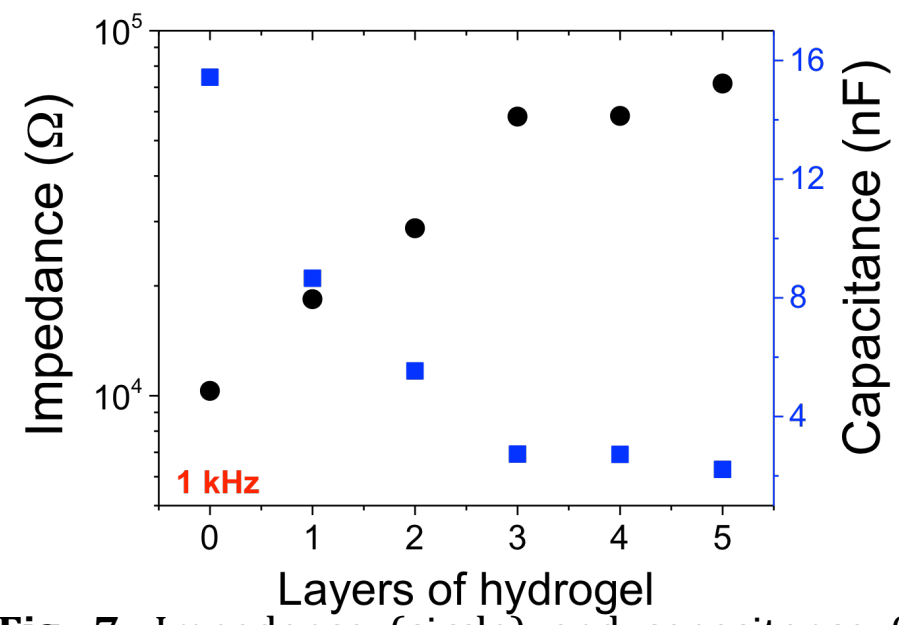

Fig. 7. Impedance (circle) and capacitance (square) measurements for increasing numbers of hydrogel layers on top of the electrode, measured in DI water. 
(A) Glucose

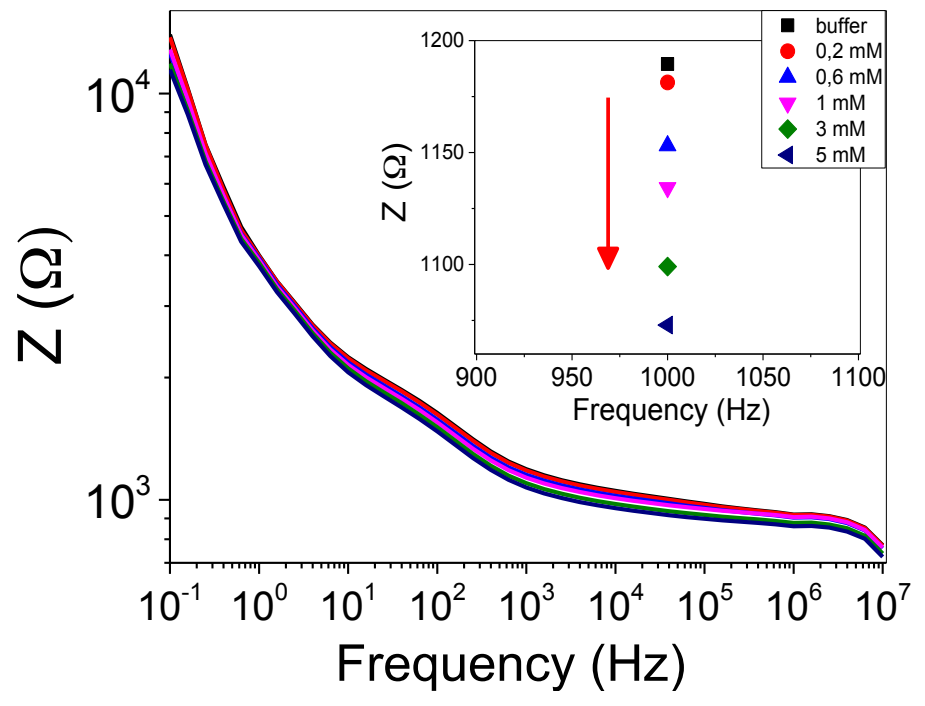

(B) Fructose

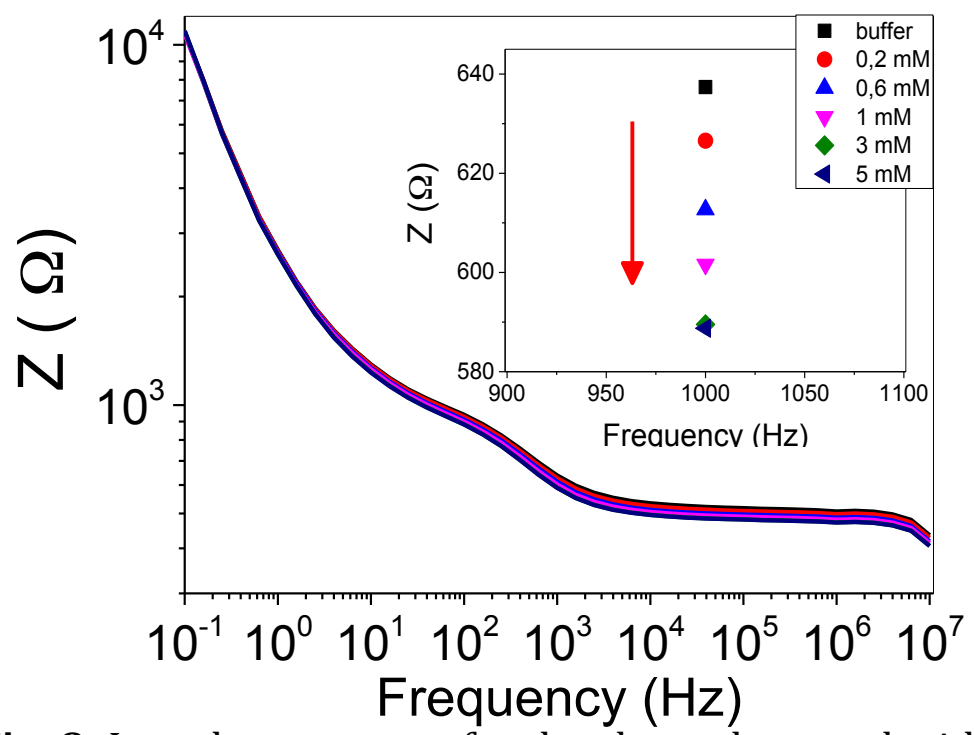

Fig. 8. Impedance spectra for the electrode coated with one layer of p(AAm-CoPBA) hydrogel with 20 mol\% PBA when exposed to varied concentrations of glucose $(A)$ and fructose $(B)$, respectively. 

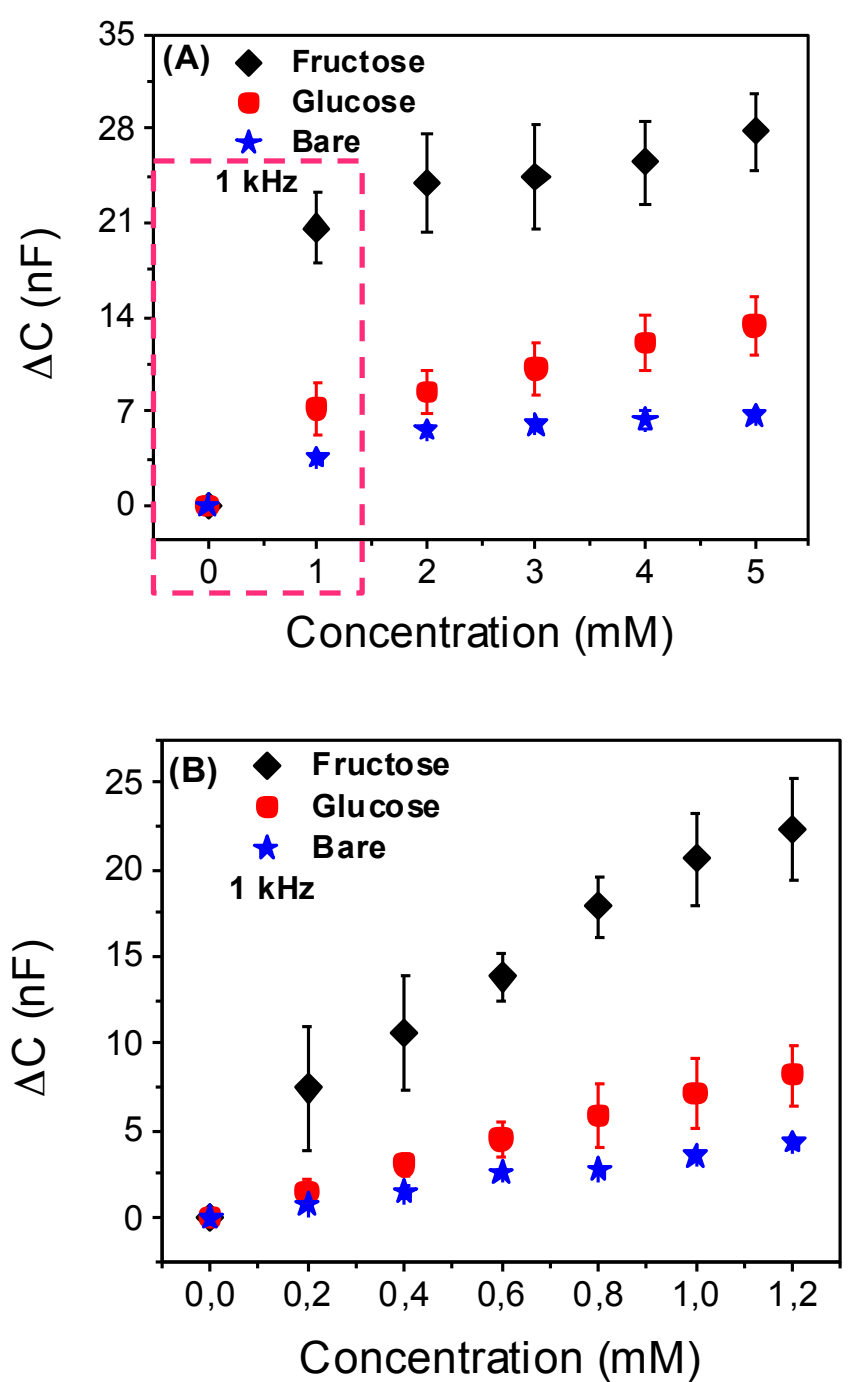

Fig. 9. Capacitance changes $(\Delta \mathrm{C})$ at $1 \mathrm{kHz}$ for the electrode coated with $\mathrm{p}$ (AAmco-PBA) hydrogel (containing $20 \mathrm{~mol} \%$ PBA) versus sugar concentration. The data was taken in triplicate and error bars were obtained from the standard deviations. The electrode without hydrogel (star) was tested with different glucose concentrations to compare the effect of PBA on capacitance response. 